\title{
A biblioteca escolar como espaço diferenciado: a perspectiva da cultura escolar
}

\author{
The School Library as a differentiated space: the school culture perspective
}

\begin{abstract}
Andreza Ferreira Félix
Mestranda do Programa de Pós-graduação em Ciência da Informação da UFMG. Bibliotecária e Pedagoga, professora da educação básica na rede privada de Belo Horizonte - MG, Brasil.

E-mail: andreza.felix@gmail.com

Adriana Bogliolo Sirihal Duarte

Doutor em Ciência da Informação pela Universidade Federal de Minas Gerais - UFMG. Profa. adjunta da Universidade Federal de Minas Gerais - UFMG.

E-mail: bogliolo@eci.ufmg.br
\end{abstract}

\section{Resumo}

Este artigo é resultado de pesquisa conduzida no Programa de Pós-graduação em Ciência da Informação da Universidade Federal de Minas Gerais - PGCI-UFMG. A pesquisa trata das práticas educativas desenvolvidas em bibliotecas escolares da Rede Municipal de Educação de Belo Horizonte relacionando-as à cultura escolar. É apresentado o conceito de bibliotecas efetivas enquanto espaços que ofertam condições de acesso ao acervo bibliográfico mas que também se constituem como espaços de aprendizagem. A pesquisa teve como objetivo principal discutir qual a natureza da cultura escolar que favorece bibliotecas efetivas. Esta questão norteou o percurso da pesquisa buscando diagnosticar e analisar práticas educativas em bibliotecas escolares e sua relação a cultura escolar abarcando elementos como: como o ambiente e no qual são produzidas e os sujeitos que as produzem. A amostra foi composta de três escolas da região metropolitana de Belo Horizonte na qual participaram diretores, bibliotecários e dois professores em cada instituição. Os dados coletados foram transcritos e categorizados para análise qualitativa, de vertente intepretativa. Os resultados da pesquisa apontam para aspectos conceituais do termo cultura escolar e analisa as práticas do sujeitos como foco para entender como a biblioteca se constitui enquanto um espaço diferente da sala de aula, tais práticas e relações figuram como objeto principal deste artigo. Evidencia como esta disposição contribui positivamente para que a biblioteca exerça seu papel educativo. Conclui que a cultura escolar é uma importante dimensão de análise para compreensão da função educativa da biblioteca.

Palavras-chave: bibliotecas escolares, biblioteca efetiva, cultura escolar, espaço diferenciado, papel educativo

\begin{abstract}
This paper have the findings of the research conducted in PPGCI-UFMG and addresses to the relationship between school library educational practices and its relationship with the school culture. The research was conducted in brazilian municipal schools, in Belo Horizonte city. The research aimed to discuss what was the nature of the school culture that supports a sucessful library program. The goal was to diagnose and analyze educational practices in brazilian school libraries and its relationship with school culture in the following aspect: how the environment affects and produces behaviors and actions related to the school library. The sample was composed by three school libraries, from public school in the city of Belo Horizonte. Principals, school librarians and two teachers - in each school - were interviewed. The data was collected using semi-structured surveys, characterizing the study as a qualitative in-depth research. Three important findings shown the relationship between school library and school culture: the presence of a collaborative culture, a specific school structure that supports the library and the school library as a differentiated space. There are aspects in this difference that contribute to the establishment of an effective school library. These factors are highlighted as: the school library as a connector, the distinctions between school libraries and classrooms and the school library location. The conclusion is that the school culture is a key dimension in school libraries activities.
\end{abstract}

Keywords: school libraries school culture, effective libraries, school culture, school library location. 


\section{Introdução}

Este artigo é parte dos resultados de pesquisa desenvolvida no Programa de PósGraduação em Ciência da Informação da Escola de Ciência da Informação da UFMG. Atualmente observamos que a biblioteca escolar é alvo de um novo olhar sobre seu papel dentro da Educação. Recentemente há um movimento no sentido de reconhecer e valorizar a função educativa da biblioteca, ampliando a perspectiva de atuação de seus profissionais e também de pesquisadores. Sempre contemplada na literatura como um espaço privilegiado da formação de leitores hoje a biblioteca escolar é analisada pelo paradigma da aprendizagem.

Experiências bem sucedidas em bibliotecas escolares podem estar ligadas a diversos fatores. Alguns destes fatores podem estar relacionados à características específicas de bibliotecas escolares que favorecem a colaboração e promovem projetos didáticos, construindo nestes espaços situações reais de aprendizagem (ALEXANDERSON; LIMBERG, 2003). Acredita-se ser de fundamental importância refletir sobre como vêm sendo conduzidas ações educativas em bibliotecas escolares brasileiras e compreender de que modo os elementos da cultura escolar (disposições, projetos, corpo docente, verbas e outros artefatos, valores e fundamentos) se relacionam com tais experiência. Entende-se, ainda, que a análise mais detalhada de bibliotecas efetivas - a partir da perspectiva dos sujeitos bibliotecários, diretores e professores - concorra para um fortalecimento das pesquisas do campo do papel educativo da biblioteca. Tal compreensão articulada com o contexto escolar em que cada biblioteca está inserida é também uma abordagem pouco explorada em pesquisas do campo (HOWARD, 2010).

Um grande corpo de pesquisadores - sobretudo nos Estados Unidos - dedica seus estudos na primeira década dos anos 2000 em compreender, diagnosticar e justificar a importância das bibliotecas escolares sob essa ótica. Alguns dos principais estudos conduzidos comprovaram, a partir da proposta metodológica da prática baseada em evidência (TODD, 2009), que as pesquisas sobre o campo forneceriam importantes subsídios para bibliotecários e gestores de políticas públicas melhor conduzirem seus projetos e gastos com a biblioteca escolar. Dentre os principais estudos destacamos os conduzidos pelo pesquisador americano Keith Lance nos anos 2000, 2005 e 2010, no estado norte-americano do Colorado e o de Ross Todd e Carol Kuhthau realizado em 2004, também nos Estados Unidos, no estado de Ohio. Os principais resultados destas pesquisas evidenciaram que bibliotecas escolares são importantes e que contribuem para um melhor desempenho dos alunos. 
Outro conceito importante que advém desta perspectiva é conceito de biblioteca efetiva. Este conceito foi melhor definido por Lance, Rodney, Hamilton-Pennell (2000) que elenca alguns elementos constituintes de uma biblioteca efetiva: é uma biblioteca que possui profissional bibliotecário, possui acesso à tecnologia, tem acervo adequado e diversificado, tem horário de funcionamento flexível e investe valores monetários na manutenção e ampliação do espaço e acervo. Uma das definições apontou: “(...) biblioteca efetiva é aquela que sob a responsabilidade de um bibliotecário que tem clareza de seu papel educativo e que desempenha um papel fundamental na aprendizagem e na construção do conhecimento" (TODD; KUHTLHAU, 2004, p. 13). Este conceito, construído a partir de usos que os estudantes faziam da biblioteca e de sua percepção sobre o impacto da biblioteca escolar sobre sua própria aprendizagem trouxe um novo olhar sobre o espaço da biblioteca e sua função dentro da escola. Ainda segundo o pesquisador: "uma biblioteca efetiva é não somente um local onde está a informação mas um espaço do conhecimento, em que os estudantes desenvolvem a cultura da aprendizagem, usando a informação para construir conhecimento" (TODD; KUHTLTHAU, 2004, p. 6). Campello também aponta uma definição para bibliotecas efetivas:

\footnotetext{
Boas bibliotecas propiciam uma aprendizagem peculiar, diferente daquela em que o aluno é um recipiente passivo de informações passadas pelo professor. É uma aprendizagem em que o estudante constrói seu conhecimento, explorando um vasto repertório de experiências vividas e registradas por outros, extraindo deles significados e agregando suas próprias experiências. (CAMPELLO, 2012, p.7)
}

A eficácia nas bibliotecas escolares, portanto, não é um conceito utópico, mas possível de ser concebido. Dentro da atual realidade das bibliotecas escolares brasileiras foram encontrados, a partir da pesquisa empírica, exemplos deste bom trabalho de biblioteca. Analisando seus projetos e a perspectiva de alguns sujeitos que promovem estas práticas, buscamos traçar uma relação entre a biblioteca escolar efetiva e a cultura escolar.

Para entender as relações entre biblioteca e cultura escolar é preciso inicialmente apresentar alguns aspectos conceituais do termo. Para pensar a cultura escolar é possível observar que o termo perpassa por vários campos do conhecimento, entre eles a antropologia, a educação, a história, a sociologia e a política. A cultura escolar pode se entendida como um conjunto de valores, ações e crenças que a escola produz, possui e reproduz em sua estrutura curricular e em sua ordem cotidiana, de fazeres do dia a dia (FARIA FILHO; VIDAL, 2004) .

Pensar a biblioteca sobre o viés da cultura escolar é pensar sobretudo a lógica de crenças e atitudes que alguns dos sujeitos desta cultura - professores, diretores e 
A biblioteca escolar como espaço diferenciado: a perspectiva da cultura escolar

bibliotecários - reproduzem no cotidiano que determinam o modo com a escola organiza seu projeto educativo. Aqui falamos de que valores e sobretudo das práticas que a escola profere, por quais deles se organiza e se mobiliza (TODD; GORDON; LU, 2011). A cultura escolar trata das práticas dos sujeitos e como, a partir destas, o cotidiano se constrói e se reconstrói. Segundo Michel de Certeau (2000, p. 100) "conhecer as apropriações feitas pelos atores no cotidiano da escola é produzir uma história das práticas escolares e, portanto, compreender a produção da cultura escolar." Assim, a cultura escolar envolve toda a vida escolar, todo o fazer que se constrói na prática dos sujeitos, na manipulação e através do cotidiano. A cultura escolar expressa crenças e valores sobre como a escola deles se utilizam para definir a aprendizagem, sobretudo aquela que se dá fora dos ambientes tradicionais de ensino.

Neste sentido observamos como alguns elementos do cotidiano, dos modos de fazer de seus valores, e ações mobilizados pelos produtores dos processos educativos podem impactar positivamente para o estabelecimento de uma biblioteca eficaz.

A análise das relações entre cultura escolar e biblioteca, realizada a partir de pesquisa empírica conduzida em 2014/2014, revelou a interconexão de vários elementos. Neste artigo destacaremos o status de espaço diferenciado que a biblioteca escolar assume quando desempenha seu papel educativo de forma eficaz. Segundo Todd, Gordon e Lu (2011) a biblioteca se constitui de diversas formas como um espaço distinto da sala de aula. As implicações desta distinção revelam não somente práticas dos sujeitos mas também modos de fazer, valores e ações representando um patrimônio simbólico que nos ajuda a pensar a cultura escolar como campo de investigação para a função educativa da biblioteca escolar. Neste caso, interessa saber como a cultura escolar se articula com os saberes que se constroem e com as práticas de aprendizagem desenvolvidas na biblioteca escolar. 


\section{O papel educativo da biblioteca}

Capacitar e preparar as pessoas para utilizar a biblioteca e seus recursos tem sido uma das preocupações dos bibliotecários em relação a seus usuários. Uma das origens, portanto, do papel educativo da biblioteca escolar foram as pesquisas acerca da educação e treinamento de usuários. No Brasil o interesse pelos estudos de educação de usuários teve seu início nos fins da década de 1990, nas bibliotecas universitárias. O desenvolvimento destes estudos mostrou que e a biblioteca e o bibliotecário desempenhavam um papel educativo ao treinar usuários para acessar os catálogos e para manipular as fontes bibliográficas. A ideia de treinar usuários evoluiu para a organização de serviços como guias, folhetos e um aprimoramento das funções do chamado serviço de referência (OTA, 1990).

Atualmente com o crescimento do volume e a ampliação dos suportes da informação, sobretudo os ligados à tecnologia novas modalidades de mediação entre informação e usuário surgem. Este cenário tem impacto nas atividades das bibliotecas e abre espaço para novas práticas educativas nas bibliotecas, inclusive as escolares (CAMPELLO, 2009a). O letramento informacional (informational literacy) surge neste sentido em interface com a educação como também uma das áreas em que o papel educativo da biblioteca pode ser exercido. O letramento informacional pode ser conceituado como uma capacidade dos cidadãos de se adaptar à cultura digital e à globalização implicando a uma capacidade das pessoas em entender, localizar, selecionar e interpretar informações de forma crítica (CAMPELLO, 2009a).

Segundo Dudziak (2003, p. 25), "a information literacy pode ser definida como um modo de aprender"; neste sentido, a biblioteca escolar passa a ser vista como um lugar que possui mais do que acervo e serviços para a educação de usuários, mas como um espaço em que se articulam ferramentas e recursos para a construção do conhecimento. Um conhecimento que, segundo a autora, é construído "a partir da busca e uso da informação, de maneira integrada ao currículo, cuja filosofia via a biblioteca como elemento chave na educação". (DUDZIAK, 2003, p. 26).

O bibliotecário coloca-se neste sentido em posição de atuar em sentidos mais amplos do treinamento como o planejamento e atividades de colaboração com professores. Outro movimento que ocorre é a demanda por serviços mais articulados às propostas curriculares também no esteio de uma valorização do profissional bibliotecário nas escolas. $\mathrm{O}$ 
A biblioteca escolar como espaço diferenciado: a perspectiva da cultura escolar

bibliotecário pode buscar uma participação mais ativa no processo educacional buscando, por exemplo, parcerias com professores. Bibliotecários e professores aplicam estratégias de modo a possibilitar o acesso, as habilidades que se acomodam ao estilo de aprendizagem de cada aluno, respeitando seu tempo e possibilidades para lidar com competências que lhe permitam analisar, sintetizar, interpretar e organizar informações em um ambiente complexo e rico em fontes de informação e recursos (CAMPELLO, 2009b).

Tais perspectivas apontam para uma ideia de que a biblioteca escolar pode, portanto, exercer seu papel educativo a partir de suas fontes, de seus profissionais, recursos tecnológicos, mas também a partir do modo como sumariza, classifica e ordena o conhecimento.

A colaboração entre professores e bibliotecários é uma das formas em que o papel educativo da biblioteca se evidencia. A colaboração é uma modalidade de educação cooperativa em que bibliotecários interagem com a equipe pedagógica, produzindo trabalhos, projetos e serviços em parceria. A colaboração é uma importante atividade que coaduna com a visão pedagógica mais participativa e com teorias educacionais (construtivismo e sóciointeracionismo) em que as principais perscpectivas apontam para o fato de que a aprendizagem se dá a partir da interação com os pares; através da expansão de conceitos e com a aprendizagem a partir do questionamento (inquiry process) (MONTIEL-OVERALL, 2005).

O papel educativo da biblioteca tem sido objeto de diversos estudos e um dos a spectos que emerge como interesse de investigação é o contexto em que cada biblioteca está inserida como uma dimensão chave nas atividades e projetos da biblioteca escolar (HOWARD, 2010) . 


\subsection{Percurso metodológico: bibliotecas escolares efetivas}

O percurso metodológico da pesquisa adotou alguns recortes conceituais importantes. Um destes recortes conceituais é o, já previamente discutido, conceito de biblioteca efetiva. $\mathrm{O}$ outro é a escolha Programa de Bibliotecas da Rede Municipal de Educação de Belo Horizonte, para efeitos práticos, chamado no texto de PB-RMEBH. O PB-RMEBH surge inicialmente como um programa de revitalização das bibliotecas escolares de Belo Horizonte, dentro do contexto da Escola Plural.

O parâmetro para a escolha das instituições pesquisadas foi feito a partir do diagnóstico da ação pedagógica efetiva na biblioteca de cada escola. Para o estabelecimento deste parâmetro foi utilizada a escala de níveis de colaboração estabelecida por MontielOverall (2005). A autora estabelece quatro níveis crescentes de colaboração entre professores e bibliotecários para a aprendizagem, que nos fornecerão um modelo para a seleção dos participantes da pesquisa: coordenação, cooperação, instrução integrada e currículo integrado

A amostra foi composta de três escolas da rede municipal de educação de Belo Horizonte e foram selecionados três principais sujeitos para tomarem parte de entrevistas semi-estruturadas: diretores, professores e bibliotecários A partir da transcrição de seus relatos, coleta de documentos pedagógicos das escolas e análise da revisão de literatura foram organizados os principais resultados a partir de categorias de análise, compostas objetivando analisar a relação entre cultura escolar e biblioteca nos espaços escolares.

\section{A biblioteca escolar enquanto espaço diferenciado}

A cultura escolar, como um conjunto de valores, ações e crenças, pode nos ajudar a pensar as dinâmicas que envolvem escola, educação e biblioteca. Uma destas dinâmicas é a apresentação da biblioteca enquanto um espaço diferente da sala de aula. Os sujeitos investigados na pesquisa aqui descrita - professores, diretores e bibliotecários - revelaram, através de suas práticas e projetos, como esta distinção se consolida como um valor importante na atuação da biblioteca ao cumprir sua função educativa.

Professores, diretores e bibliotecários pesquisados em três escolas da Região Metropolitana de Belo Horizonte, parte do Programa de Bibliotecas da Prefeitura Municipal de Belo Horizonte relataram suas relações, parcerias e percepções acerca de suas bibliotecas 
A biblioteca escolar como espaço diferenciado: a perspectiva da cultura escolar

escolares. Estas bibliotecas foram escolhidas por representarem uma amostra do que se chamou de "biblioteca efetiva", ou seja, uma biblioteca que atende aos critérios apresentados sobre este conceito mas, também, em que se verificou a existência de projetos de colaboração entre professores e bibliotecários.

A avaliação da biblioteca escolar como espaço diferenciado revelou as seguintes categorias de análise: a biblioteca como espaço de conexões (conexões entre os próprios usuários, conexões entre a biblioteca e os professores, conexões entre a biblioteca e os alunos); a biblioteca como espaço distinto da sala de aula (organização espacial, mediação) e a localização da biblioteca. Cada uma das categorias será apresentada a seguir.

\subsection{A biblioteca como um espaço de conexões}

Conforme Todd, Gordon e Lu, a biblioteca é um lugar de conexões. Para os autores, "a biblioteca escolar oferece mais escolhas do que uma sala de aula em que o ambiente informacional é estreitado por seu escopo" (TODD; GORDON; LU, 2011, p. 58).

Os resultados revelaram que o ambiente, os valores e as práticas em que cada escola está inserida têm grande influência sobre o trabalho que a biblioteca escolar desempenha. A biblioteca, segundo os participantes desta pesquisa, se constitui muitas vezes como um local em que o acesso é permanente e em que a socialização e o convívio se dão de forma mais relaxada e menos controlada do que em sala de aula. As relações entre os alunos são mais horizontalizadas e vários alunos de idades diversas estão em contato constante em uma interação menos mediada pelo currículo. Assim relata um dos professores entrevistados na pesquisa empírica:

É como se fosse um ponto de passeio também. Eu observo lá na biblioteca, por exemplo, os meninos do ensino fundamental 2 batendo papo, lendo jornal, trocando ideia com os livros pra idade deles. (Relato de professor entrevistado).

Além dos processos de socialização e convívio, uma outra perspectiva de conexões que a biblioteca estabelece com seus usuários e com a comunidade escolar é aquela entre a biblioteca e a formação de professores. Os professores reconhecem que o acervo e os serviços da biblioteca ajudam, possibilitando a produção de trabalhos melhores do que sem a biblioteca. 
Andreza Ferreira Félix e Adriana Bogliolo Sirihal Duarte

Nossa! Eu não trabalharia se não fosse a biblioteca. Eu vou lá todos os dias. A nossa biblioteca tem desde de livros didático-pedagógicos, coleções, a cds. Você consegue montar o seu projeto todo dentro da biblioteca. (Relato de professor entrevistado).

A organização mais flexível da rotina da biblioteca tem impacto positivo no acesso dos alunos. As regras e a organização do espaço estabelecem conexões positivas com os alunos na medida em que ofertam organização com padrões específicos mas com uma mediação com um controle diferente do que se estabelece na relação sala de aula-aluno.

A biblioteca estabelece com seus alunos conexões inclusive de natureza afetivopessoal. Estas conexões se estabelecem tanto quando o bibliotecário e funcionários da biblioteca interagem com os alunos, quanto quando o acervo bibliográfico, projetos e recursos tem impacto pessoal na formação dos mesmos.

\footnotetext{
Aqui a biblioteca é dos meninos. O público em geral precisa se sentir em casa na biblioteca. (Relato de professor entrevistado).

Nem que fosse um aconchego, um afago no coração. Porque tem muito aluno que a gente vê que não recebe carinho de forma alguma. Às vezes o bom dia que o menino vai ouvir é o bom dia que eu dou. Eu recebo os meus alunos com um atendimento humanizado, caloroso, que é importante. (Relato de bibliotecário entrevistado).
}

Embora este seja um fator que raramente esteja submetido a avaliações mais formais, as conexões que se estabelecem entre biblioteca e usuário, quando afetivas, tendem a afetar positivamente a percepção que ambos tem sobre a importância e eficácia da biblioteca.

Faria Filho e Vidal (2004) afirma que o olhar da cultura escolar nos permite perceber que a cultura produz os sujeitos mas que também eles são produzidos por estas mesmas práticas; sendo assim, as relações pessoais que se estabelecem na biblioteca escolar afetam tanto os sujeitos quanto a própria cultura escolar que as produziu. Em uma biblioteca escolar em que há diversos tipos de conexões viabilizadas pelas práticas dos sujeitos, seu papel junto a comunidade escolar é ampliado, como também é a percepção de que ela é importante para a educação e para a escola. 
A biblioteca escolar como espaço diferenciado: a perspectiva da cultura escolar

\subsection{A biblioteca como um espaço distinto da sala de aula}

A biblioteca escolar tem geralmente uma sala própria; o ideal é que tenha mobiliário específico, computadores para funcionários e alunos, mesas coletivas, mesas individuais e acondicionamentos para material bibliográfico diferenciado, apresente acervo catalogado e possua um funcionário responsável. Estes são, segundo Campello (2011), alguns dos principais parâmetros para bibliotecas escolares. Observando a descrição, é possível demarcar claras diferenças da biblioteca escolar para uma sala de aula, inclusive em sua organização espacial, disposição dos objetos e layout dos equipamentos. A biblioteca é, em termos estruturais, diferente de uma sala de aula.

Para além das distinções físicas, a biblioteca escolar é um lugar de práticas culturais distintas da sala de aula. Lance (2005) destacam o impacto positivo do horário flexível de atendimento da biblioteca; de modo que ela está acessível em tempos que não são delimitados pelo horário curricular da escola. A biblioteca pode ser acessada por alunos em turnos diferentes, em horários e de formas distintas do que outros espaços escolares. Francis, Lace e Lietzau (2010) comprovam em estudo mais recente alunos que tem na biblioteca escolar um espaço a que podem acessar livremente tendem a ter melhor desempenho escolar porque, em grande medida, sua estrutura e organização distintas da sala de aula atrai os alunos para um uso mais livre dos recursos informacionais e do espaço físico.

$\mathrm{Na}$ pesquisa empírica isso foi identificado e pode ser ilustrado, por exemplo, no seguinte depoimento:

\footnotetext{
Eles gostam de ler, eles gostam desse espaço, muito gostoso, aconchegante, acolhedor. Aqui é um ambiente acolhedor mesmo, você gosta, você vê, você pega um livro. A organização, a disposição deles, acho que isso tudo faz com que os alunos sejam estimulados a ler, a buscar. A marca dessa biblioteca é o aconchego. Às vezes, quando a gente está atribulado, a gente vem aqui. As auxiliares, até quando estão apertadas e a biblioteca está cheia, dão atenção para a gente. Isso faz com que a gente se sinta bem. Elas tem uma autonomia própria delas, do cargo delas, que faz tudo funcionar bem, todo dia, e que está muito em sintonia com o trabalho do resto da escola. (Relato de professor entrevistado).
}

A biblioteca atua como um espaço para aprimoramento pessoal. Neste sentido a biblioteca assume um importante papel em dar suporte à leitura autônoma quando ofertada em condições diferentes das curriculares, tendendo a aumentar os níveis de acesso à cultura escrita. Justamente porque o acesso à leitura e a relação dos alunos com o livro literário é intermediada por atitudes diferentes das promovidas pela escola, em que, muitas vezes a relação com a leitura literária é mediada por práticas escolarizadoras e avaliativas. 
Andreza Ferreira Félix e Adriana Bogliolo Sirihal Duarte

Essa relação que eu tenho com a cultura, com o conhecimento, fez eu abrir o meu olhar para a biblioteca. Eu gosto muito de ler, eu gosto muito de biblioteca, eu vivo com um livro na bolsa. A escola está sempre incentivando. Eu tenho um poema meu publicado aqui no livro da escola. Quando eu levei para casa meus filhos ficaram emocionados. Tem muitos projetos aqui. Eu gosto muito do acervo. (Relato de professor entrevistado).

Então, eu acredito que o sucesso é essa questão mesmo da biblioteca fazer parte da escola e não ser como antigamente a gente via, só um lugar de silêncio, de empréstimo de livro. Por exemplo no recreio, a gente usa muito a biblioteca. (...) É um espaço educador. E não só de leitura e de empréstimo, né? (Relato de diretor entrevistado).

O sentido de biblioteca como lugar do silêncio, da guarda, do isolamento; uma visão que marcou a biblioteca escolar é transformado por uma cultura em que o foco principal é a aprendizagem dos alunos. Esta mudança de abordagem ocasiona uma série de desdobramentos nos valores e nas atividades escolares e amplia o olhar sobre a biblioteca escolar e suas possibilidades de contribuição e participação neste novo cenário educativo.

\subsection{A localização da biblioteca}

Considerando que a cultura escolar abarca dimensões do cotidiano da escola, pode-se afirmar que os tempos e também os seus espaços representam vivamente as práticas dos sujeitos dentro da comunidade escolar. Ao pensar nos espaços vemos que a localização da biblioteca surge como um elemento importante para que seu trabalho tenha visibilidade e também reconhecimento. Quando a biblioteca escolar está localizada em local privilegiado dentro do espaço da escola, está mais acessível, aberta e visível para seus usuários. De modo geral o local privilegiado é no prédio principal ou perto do pátio no qual a maior parte dos alunos, professores, diretores, funcionários e outros membros da escola precisa transitar diariamente.

Em posição central, ou privilegiada, os sujeitos escolares têm mais clareza da atuação da biblioteca no cotidiano. Os usuários reconhecem que esta localização garante visibilidade e confiança, ou um sentimento de que os usuários podem contar com a biblioteca, o que segundo Lance, Rodney e Russell (2007) gera um sentimento de pertencimento. A biblioteca escolar localizada em posição central, portanto, deixa de estar isolada em projetos e serviços que nada têm a ver com a dinâmica escolar e passa a ser vista como parte integrante do aparato escolar, o que é fundamental para que estabeleçam, por exemplo, atividades de colaboração com professores. 
A biblioteca escolar como espaço diferenciado: a perspectiva da cultura escolar

Quando a biblioteca se coloca à disposição dos alunos apresentando suas instalações mais centralizadas está também mais disponível para oferecer possibilidades educativas diversificadas, o que pode favorecer o aluno em seu contato, acesso e manipulação de novas fontes de informação, beneficiando a construção do conhecimento (CAMPELLO, 2012).

Nas escolas investigadas, as bibliotecas ocupavam sempre localização privilegiada. E isso não parecia ser mera coincidência, mas também parte de uma cultura escolar. Os entrevistados demonstraram ter consciência dessa condição:

Eu já percebi, assim, nessa escola, o fato de a biblioteca ser centralizada, todo mundo que entra na escola passa pela biblioteca. A localização dessa biblioteca, nessa escola, é de extrema importância, não tem nenhum aluno que não tenha entrado na biblioteca ou que não entre lá, nem que seja pra falar oi. (Relato de professor entrevistado).

\section{Conclusões}

Pensar a biblioteca escolar e seu potencial educativo sob a ótica da cultura escolar foi o objetivo principal deste artigo. O ponto de partida foi o conceito de bibliotecas efetivas concentrando-se em destacar bibliotecas que ofertavam bons serviços. Bibliotecas escolares que, possuindo profissionais bibliotecários e fazendo parte de um programa de bibliotecas fossem bem sucedidas em implementar a ação pedagógica. A análise destes fatores revelou que não somente a cultura escolar pode influenciar a condução de um bom trabalho de biblioteca mas que parte de sua eficácia advém do fato de a biblioteca escolar se constituir enquanto um espaço diferente da sala de aula.

Se constituir como um espaço diferenciado significa situar a biblioteca como um espaço de conexões. A conectividade que a biblioteca exerce com seus usuários é diferente da relação social e afetiva que se estabelece em sala de aula. Esta relação parece fortalecer a importância da biblioteca como um espaço independente e contribuinte para o projeto educacional de cada escola. Destaca-se ainda a importância que a localização da biblioteca tem na sua visibilidade junto à comunidade escolar; fazendo do diálogo e do compartilhamento de ideias e experiências também uma questão arquitetônica. A potencialidade da biblioteca como um lugar onde se pode obter mais dados e ampliar as experiências de aprendizagem também demarcam sua função como espaço diferenciado.

As práticas do cotidiano entre os sujeitos na escola tem muitos elementos a fornecer no debate sobre o papel educativo da biblioteca. Evidencia a existência de elementos que vão 
além do discurso e que se fundamentam na realidade da biblioteca nas relações que se estabelecem entre biblioteca escolar e comunidade escolar.

\section{Referências}

ALEXANDERSSON, M.; LIMBERG, L. The school library as a space for learning. School Library Worldwide, v. 9, n. 1, p. 1-18, 2003.

CAMPELLO, B. Biblioteca escolar: conhecimentos que sustentam a prática. Belo Horizonte: Autêntica, 2012.

Letramento informacional no Brasil: práticas educativas de bibliotecários do ensino básico. 2009. 209 f. Tese (Doutorado em Ciência da Informação) - Escola de Ciência da Informação, Unversidade Federal de Minas Gerais, Belo Horizonte, 2009a .

- Letramento informacional: função educativa do bibliotecário na escola. Belo Horizonte: Autêntica, 2009b.

Parâmetros para bibliotecas escolares brasileiras: fundamentos de sua elaboração. Informação e Sociedade, João Pessoa, v. 21, p 105-120, maio/ago. 2011.

CERTEAU, M. A invenção do cotidiano: artes de fazer. Petrópolis: Vozes, 2000.

DUDZIAK, E. A. Information literacy: princípios, filosofia e prática. Ciência da Informação, Brasília, v. 32, n. 1, p. 23-35, jan./abr. 2003.

FARIA FILHO, L. M.; VIDAL, D. G. A cultura escolar como categoria de analise e como campo de investigação na história da educação. Educação e Pesquisa, São Paulo, v. 30. p 139-159, jan./abr. 2004.

FRANCIS, B. H.; LANCE, K. C.; LIETZAU, Z. School librarians continue to help students achieve standards: The third Colorado study (2010). A Closer Look, p. 1-20, 2010.

HOWARD, J. K. The relationship between school culture and the school library program: four case studies. American Library Assotiation, v. 13, p 1-14, out. 2010.

LANCE, K. C. Powerful libraries make powerful learners: the Illinois study. 2005. .; RODNEY, M. J.; RUSSELL, B. How students, teachers \& principals benefit from strong school libraries: the Indiana study. Association for Indiana Media Educators, 2007.

.; ___ _ HAMILTON-PENNELL, C. How School librarians help kids achieve standards. the second Colororado study. A Closer View, 2000.

MONTIEL-OVERALL, P. A theoretical understanding of teacher and librarian collaboration (TLC). School libraries worldwide, v. 11, n. 2, p 24-48, jul. 2005. 
OTA, M. E. C. Educação de usuários em bibliotecas universitárias brasileiras:

revisão de literatura nacional. Revista de Biblioteconomia e Documentação, v. 23, n. 1/4, p. 38-57, jan./dez. 1990.

TODD, R. J.; KUHLTHAU, C. Student learning through Ohio school libraries:

background, methodology and report of findings. Columbus: OELMA, 2004.

; GORDON, C.; LU, Y. Report on findings and recommendations of the New

Jersey school library study phase 2: once common goal: student learning. Rutgers

University: CISSL, 2011. 181 p.

School librarianship and evidence based practice: progress, perspectives, and

challenges. Evidence Based Library and Information Practice, v. 4, n. 2, p. 78-96, 2009. 